\title{
72-h diurnal RNA-seq analysis of fully expanded third leaves from maize, sorghum, and foxtail millet at 3-h resolution
}

\author{
Xianjun Lai ${ }^{1,2 \dagger}$, Claire Bendix ${ }^{3 \dagger}$, Yang Zhang ${ }^{1}$, James C. Schnable ${ }^{1}$ and Frank G. Harmon ${ }^{4,3^{*}}$ (1)
}

\begin{abstract}
Objectives: The purpose of this data set is to capture the complete diurnal (i.e., daily) transcriptome of fully expanded third leaves from the C4 panacoid grasses sorghum (Sorghum bicolor), maize (Zea mays), and foxtail millet (Setaria italica) with RNA-seq transcriptome profiling. These data are the cornerstone of a larger project that examined the conservation and divergence of gene expression networks within these crop plants. This data set focuses on temporal changes in gene expression to identify the network architecture responsible for daily regulation of plant growth and metabolic activities. The power of this data set is fine temporal resolution combined with continuous sampling over multiple days.

Data description: The data set is 72 individual RNA-seq samples representing 24 time course samples each for sorghum, maize, and foxtail millet plants cultivated in a growth chamber under equal intervals of light and darkness. The 24 samples are separated by 3-h intervals so that the data set is a fine scale 72-h analysis of gene expression in the leaves of each plant type. FASTQ files from Illumina sequencing are available at the National Center for Biotechnology Information Sequence Read Archive.
\end{abstract}

Keywords: Diurnal rhythms, Fastq file, Foxtail millet, Maize, Panacoid grasses, Poaceae; RNA-seq, Sorghum

\section{Objective}

The purpose of this data set is to identify transcripts with diurnal (i.e. daily) patterns of regulation within the $\mathrm{C} 4$ panacoid grasses sorghum (Sorghum bicolor), maize (Zea mays), and foxtail millet (Setaria italica) with RNA-seq transcriptome profiling. These data are the cornerstone of a larger project that examined the conservation and divergence between sorghum, maize and foxtail millet of circadian clock and diurnal gene expression networks and their local DNA regulatory elements [1]. The working hypothesis motivating the project is critical regulatory features are retained through evolution. Therefore,

*Correspondence: fharmon@berkeley.edu; frank.harmon@usda.gov

${ }^{\dagger}$ Xianjun Lai and Claire Bendix contributed equally to this work

${ }^{4}$ Plant Gene Expression Center, USDA-ARS, Albany 94710, USA

Full list of author information is available at the end of the article these features can be discovered by finding transcriptome attributes shared between related, but evolutionarily distant, plant species. The power of this data set is combined fine temporal resolution of sampling and continuous sampling over a 72-h period. Sorghum, maize, and foxtail millet are studied because each is from the panacoid family of grasses, which perform $\mathrm{C} 4$ photosynthesis, and is a major crop plant worldwide. This data set focuses on temporal changes in gene expression to identify the network architecture for daily regulation of plant growth and metabolic activities.

\section{Data description}

The data set is 72 individual RNA-seq samples (Table 1) representing 24 fully expanded third leaf samples each for sorghum (BTx623 inbred), maize (B73 inbred), and foxtail millet (Yugu1 accession) plants cultivated in a

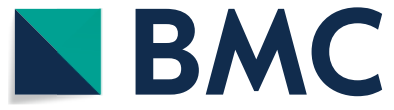

(c) The Author(s) 2021. This article is licensed under a Creative Commons Attribution 4.0 International License, which permits use, sharing, adaptation, distribution and reproduction in any medium or format, as long as you give appropriate credit to the original author(s) and the source, provide a link to the Creative Commons licence, and indicate if changes were made. The images or other third party material in this article are included in the article's Creative Commons licence, unless indicated otherwise in a credit line to the material. If material is not included in the article's Creative Commons licence and your intended use is not permitted by statutory regulation or exceeds the permitted use, you will need to obtain permission directly from the copyright holder. To view a copy of this licence, visit http://creativeco mmons.org/licenses/by/4.0/. The Creative Commons Public Domain Dedication waiver (http://creativecommons.org/publicdomain/ zero/1.0/) applies to the data made available in this article, unless otherwise stated in a credit line to the data. 
Table 1 Overview of data files/data sets

\begin{tabular}{|c|c|c|c|}
\hline Label & Name of data file/data set & $\begin{array}{l}\text { File types (file } \\
\text { extension) }\end{array}$ & Data repository and identifier (DOI or accession number) \\
\hline Data file 1 & RNA-seq of B73: fully expanded third leaves $3 \mathrm{~h}$ & fastq & $\begin{array}{l}\text { NCBI Sequence Read Archive (http://identifiers.org/ncbi/insdc } \\
\text {.sra:SRR1 1442908) }\end{array}$ \\
\hline Data file 2 & RNA-seq of B73: fully expanded third leaves $6 \mathrm{~h}$ & fastq & $\begin{array}{l}\text { NCBI Sequence Read Archive (http://identifiers.org/ncbi/insdc } \\
\text {.sra:SRR11442907) }\end{array}$ \\
\hline Data file 3 & RNA-seq of B73: fully expanded third leaves $9 \mathrm{~h}$ & fastq & $\begin{array}{l}\text { NCBI Sequence Read Archive (http://identifiers.org/ncbi/insdc } \\
\text {.sra:SRR1 1442896) }\end{array}$ \\
\hline Data file 4 & RNA-seq of B73: fully expanded third leaves $12 \mathrm{~h}$ & fastq & $\begin{array}{l}\text { NCBI Sequence Read Archive (http://identifiers.org/ncbi/insdc } \\
\text {.sra:SRR1 1442885) }\end{array}$ \\
\hline Data file 5 & RNA-seq of B73: fully expanded third leaves $15 \mathrm{~h}$ & fastq & $\begin{array}{l}\text { NCBI Sequence Read Archive (http://identifiers.org/ncbi/insdc } \\
\text {.sra:SRR11442874) }\end{array}$ \\
\hline Data file 6 & RNA-seq of B73: fully expanded third leaves $18 \mathrm{~h}$ & fastq & $\begin{array}{l}\text { NCBI Sequence Read Archive (http://identifiers.org/ncbi/insdc } \\
\text {.sra:SRR11442859) }\end{array}$ \\
\hline Data file 7 & RNA-seq of B73: fully expanded third leaves $21 \mathrm{~h}$ & fastq & $\begin{array}{l}\text { NCBI Sequence Read Archive (http://identifiers.org/ncbi/insdc } \\
\text {.sra:SRR11442848) }\end{array}$ \\
\hline Data file 8 & RNA-seq of B73: fully expanded third leaves $24 \mathrm{~h}$ & fastq & $\begin{array}{l}\text { NCBI Sequence Read Archive (http://identifiers.org/ncbi/insdc } \\
\text {.sra:SRR1 1442837) }\end{array}$ \\
\hline Data file 9 & RNA-seq of B73: fully expanded third leaves $27 \mathrm{~h}$ & fastq & $\begin{array}{l}\text { NCBI Sequence Read Archive (http://identifiers.org/ncbi/insdc } \\
\text {.sra:SRR1 1442870) }\end{array}$ \\
\hline Data file 10 & RNA-seq of B73: fully expanded third leaves $30 \mathrm{~h}$ & fastq & $\begin{array}{l}\text { NCBI Sequence Read Archive (http://identifiers.org/ncbi/insdc } \\
\text {.sra:SRR11442869) }\end{array}$ \\
\hline Data file 11 & RNA-seq of B73: fully expanded third leaves $33 \mathrm{~h}$ & fastq & $\begin{array}{l}\text { NCBI Sequence Read Archive (http://identifiers.org/ncbi/insdc } \\
\text {.sra:SRR11442906) }\end{array}$ \\
\hline Data file 12 & RNA-seq of B73: fully expanded third leaves $36 \mathrm{~h}$ & fastq & $\begin{array}{l}\text { NCBI Sequence Read Archive (http://identifiers.org/ncbi/insdc } \\
\text {.sra:SRR11442905) }\end{array}$ \\
\hline Data file 13 & RNA-seq of B73: fully expanded third leaves $39 \mathrm{~h}$ & fastq & $\begin{array}{l}\text { NCBI Sequence Read Archive (http://identifiers.org/ncbi/insdc } \\
\text {.sra:SRR11442904) }\end{array}$ \\
\hline Data file 14 & RNA-seq of B73: fully expanded third leaves $42 \mathrm{~h}$ & fastq & $\begin{array}{l}\text { NCBI Sequence Read Archive (http://identifiers.org/ncbi/insdc } \\
\text {.sra:SRR1 1442903) }\end{array}$ \\
\hline Data file 15 & RNA-seq of B73: fully expanded third leaves $45 \mathrm{~h}$ & fastq & $\begin{array}{l}\text { NCBI Sequence Read Archive (http://identifiers.org/ncbi/insdc } \\
\text {.sra:SRR11442902) }\end{array}$ \\
\hline Data file 16 & RNA-seq of B73: fully expanded third leaves $48 \mathrm{~h}$ & fastq & $\begin{array}{l}\text { NCBI Sequence Read Archive (http://identifiers.org/ncbi/insdc } \\
\text {.sra:SRR11442901) }\end{array}$ \\
\hline Data file 17 & RNA-seq of B73: fully expanded third leaves $51 \mathrm{~h}$ & fastq & $\begin{array}{l}\text { NCBI Sequence Read Archive (http://identifiers.org/ncbi/insdc } \\
\text {.sra:SRR11442900) }\end{array}$ \\
\hline Data file 18 & RNA-seq of B73: fully expanded third leaves $54 \mathrm{~h}$ & fastq & $\begin{array}{l}\text { NCBI Sequence Read Archive (http://identifiers.org/ncbi/insdc } \\
\text {.sra:SRR11442899) }\end{array}$ \\
\hline Data file 19 & RNA-seq of B73: fully expanded third leaves $57 \mathrm{~h}$ & fastq & $\begin{array}{l}\text { NCBI Sequence Read Archive (http://identifiers.org/ncbi/insdc } \\
\text {.sra:SRR11442898) }\end{array}$ \\
\hline Data file 20 & RNA-seq of B73: fully expanded third leaves $60 \mathrm{~h}$ & fastq & $\begin{array}{l}\text { NCBI Sequence Read Archive (http://identifiers.org/ncbi/insdc } \\
\text {.sra:SRR11442897) }\end{array}$ \\
\hline Data file 21 & RNA-seq of B73: fully expanded third leaves $63 \mathrm{~h}$ & fastq & $\begin{array}{l}\text { NCBI Sequence Read Archive (http://identifiers.org/ncbi/insdc } \\
\text {.sra:SRR11442895) }\end{array}$ \\
\hline Data file 22 & RNA-seq of B73: fully expanded third leaves $66 \mathrm{~h}$ & fastq & $\begin{array}{l}\text { NCBI Sequence Read Archive (http://identifiers.org/ncbi/insdc } \\
\text {.sra:SRR1 1442894) }\end{array}$ \\
\hline Data file 23 & RNA-seq of B73: fully expanded third leaves $69 \mathrm{~h}$ & fastq & $\begin{array}{l}\text { NCBI Sequence Read Archive (http://identifiers.org/ncbi/insdc } \\
\text {.sra:SRR1 1442893) }\end{array}$ \\
\hline Data file 24 & RNA-seq of B73: fully expanded third leaves $72 \mathrm{~h}$ & fastq & $\begin{array}{l}\text { NCBI Sequence Read Archive (http://identifiers.org/ncbi/insdc } \\
\text {.sra:SRR1 1442892) }\end{array}$ \\
\hline Data file 25 & RNA-seq of Yugu1: fully expanded third leaves $3 \mathrm{~h}$ & fastq & $\begin{array}{l}\text { NCBI Sequence Read Archive (http://identifiers.org/ncbi/insdc } \\
\text {.sra:SRR1 1442861) }\end{array}$ \\
\hline Data file 26 & RNA-seq of Yugu 1: fully expanded third leaves $6 \mathrm{~h}$ & fastq & $\begin{array}{l}\text { NCBI Sequence Read Archive (http://identifiers.org/ncbi/insdc } \\
\text {.sra:SRR1 1442860) }\end{array}$ \\
\hline Data file 27 & RNA-seq of Yugu1: fully expanded third leaves $9 \mathrm{~h}$ & fastq & $\begin{array}{l}\text { NCBI Sequence Read Archive (http://identifiers.org/ncbi/insdc } \\
\text {.sra:SRR1 1442858) }\end{array}$ \\
\hline Data file 28 & RNA-seq of Yugu1: fully expanded third leaves $12 \mathrm{~h}$ & fastq & $\begin{array}{l}\text { NCBI Sequence Read Archive (http://identifiers.org/ncbi/insdc } \\
\text {.sra:SRR1 1442857) }\end{array}$ \\
\hline
\end{tabular}


Table 1 (continued)

\begin{tabular}{|c|c|c|c|}
\hline Label & Name of data file/data set & $\begin{array}{l}\text { File types (file } \\
\text { extension) }\end{array}$ & Data repository and identifier (DOI or accession number) \\
\hline Data file 29 & RNA-seq of Yugu1: fully expanded third leaves $15 \mathrm{~h}$ & fastq & $\begin{array}{l}\text { NCBI Sequence Read Archive (http://identifiers.org/ncbi/insdc } \\
\text {.sra:SRR11442856) }\end{array}$ \\
\hline Data file 30 & RNA-seq of Yugu1: fully expanded third leaves $18 \mathrm{~h}$ & fastq & $\begin{array}{l}\text { NCBI Sequence Read Archive (http://identifiers.org/ncbi/insdc } \\
\text {.sra:SRR11442855) }\end{array}$ \\
\hline Data file 31 & RNA-seq of Yugu1: fully expanded third leaves $21 \mathrm{~h}$ & fastq & $\begin{array}{l}\text { NCBI Sequence Read Archive (http://identifiers.org/ncbi/insdc } \\
\text {.sra:SRR11442854) }\end{array}$ \\
\hline Data file 32 & RNA-seq of Yugu1: fully expanded third leaves $24 \mathrm{~h}$ & fastq & $\begin{array}{l}\text { NCBI Sequence Read Archive (http://identifiers.org/ncbi/insdc } \\
\text {.sra:SRR11442853) }\end{array}$ \\
\hline Data file 33 & RNA-seq of Yugu1: fully expanded third leaves $27 \mathrm{~h}$ & fastq & $\begin{array}{l}\text { NCBI Sequence Read Archive (http://identifiers.org/ncbi/insdc } \\
\text {.sra:SRR11442852) }\end{array}$ \\
\hline Data file 34 & RNA-seq of Yugu1: fully expanded third leaves $30 \mathrm{~h}$ & fastq & $\begin{array}{l}\text { NCBI Sequence Read Archive (http://identifiers.org/ncbi/insdc } \\
\text {.sra:SRR11442851) }\end{array}$ \\
\hline Data file 35 & RNA-seq of Yugu1: fully expanded third leaves $33 \mathrm{~h}$ & fastq & $\begin{array}{l}\text { NCBI Sequence Read Archive (http://identifiers.org/ncbi/insdc } \\
\text {.sra:SRR11442850) }\end{array}$ \\
\hline Data file 36 & RNA-seq of Yugu1: fully expanded third leaves $36 \mathrm{~h}$ & fastq & $\begin{array}{l}\text { NCBI Sequence Read Archive (http://identifiers.org/ncbi/insdc } \\
\text {.sra:SRR11442849) }\end{array}$ \\
\hline Data file 37 & RNA-seq of Yugu1: fully expanded third leaves $39 \mathrm{~h}$ & fastq & $\begin{array}{l}\text { NCBI Sequence Read Archive (http://identifiers.org/ncbi/insdc } \\
\text {.sra:SRR11442847) }\end{array}$ \\
\hline Data file 38 & RNA-seq of Yugu1: fully expanded third leaves $42 \mathrm{~h}$ & fastq & $\begin{array}{l}\text { NCBI Sequence Read Archive (http://identifiers.org/ncbi/insdc } \\
\text {.sra:SRR11442846) }\end{array}$ \\
\hline Data file 39 & RNA-seq of Yugu1: fully expanded third leaves $45 \mathrm{~h}$ & fastq & $\begin{array}{l}\text { NCBI Sequence Read Archive (http://identifiers.org/ncbi/insdc } \\
\text {.sra:SRR11442845) }\end{array}$ \\
\hline Data file 40 & RNA-seq of Yugu1: fully expanded third leaves $48 \mathrm{~h}$ & fastq & $\begin{array}{l}\text { NCBI Sequence Read Archive (http://identifiers.org/ncbi/insdc } \\
\text {.sra:SRR11442844) }\end{array}$ \\
\hline Data file 41 & RNA-seq of Yugu1: fully expanded third leaves $51 \mathrm{~h}$ & fastq & $\begin{array}{l}\text { NCBI Sequence Read Archive (http://identifiers.org/ncbi/insdc } \\
\text {.sra:SRR11442843) }\end{array}$ \\
\hline Data file 42 & RNA-seq of Yugu1: fully expanded third leaves $54 \mathrm{~h}$ & fastq & $\begin{array}{l}\text { NCBI Sequence Read Archive (http://identifiers.org/ncbi/insdc } \\
\text {.sra:SRR11442842) }\end{array}$ \\
\hline Data file 43 & RNA-seq of Yugu1: fully expanded third leaves $57 \mathrm{~h}$ & fastq & $\begin{array}{l}\text { NCBI Sequence Read Archive (http://identifiers.org/ncbi/insdc } \\
\text {.sra:SRR11442841) }\end{array}$ \\
\hline Data file 44 & RNA-seq of Yugu1: fully expanded third leaves $60 \mathrm{~h}$ & fastq & $\begin{array}{l}\text { NCBI Sequence Read Archive (http://identifiers.org/ncbi/insdc } \\
\text {.sra:SRR11442840) }\end{array}$ \\
\hline Data file 45 & RNA-seq of Yugu1: fully expanded third leaves $63 \mathrm{~h}$ & fastq & $\begin{array}{l}\text { NCBI Sequence Read Archive (http://identifiers.org/ncbi/insdc } \\
\text {.sra:SRR11442839) }\end{array}$ \\
\hline Data file 46 & RNA-seq of Yugu1: fully expanded third leaves $66 \mathrm{~h}$ & fastq & $\begin{array}{l}\text { NCBI Sequence Read Archive (http://identifiers.org/ncbi/insdc } \\
\text {.sra:SRR11442838) }\end{array}$ \\
\hline Data file 47 & RNA-seq of Yugu1: fully expanded third leaves $69 \mathrm{~h}$ & fastq & $\begin{array}{l}\text { NCBI Sequence Read Archive (http://identifiers.org/ncbi/insdc } \\
\text {.sra:SRR11442872) }\end{array}$ \\
\hline Data file 48 & RNA-seq of Yugu1: fully expanded third leaves $72 \mathrm{~h}$ & fastq & $\begin{array}{l}\text { NCBI Sequence Read Archive (http://identifiers.org/ncbi/insdc } \\
\text {.sra:SRR11442871) }\end{array}$ \\
\hline Data file 49 & RNA-seq of BTx623: fully expanded third leaves $3 \mathrm{~h}$ & fastq & $\begin{array}{l}\text { NCBI Sequence Read Archive (http://identifiers.org/ncbi/insdc } \\
\text {.sra:SRR11442891) }\end{array}$ \\
\hline Data file 50 & RNA-seq of BTx623: fully expanded third leaves $6 \mathrm{~h}$ & fastq & $\begin{array}{l}\text { NCBI Sequence Read Archive (http://identifiers.org/ncbi/insdc } \\
\text {.sra:SRR11442890) }\end{array}$ \\
\hline Data file 51 & RNA-seq of BTx623: fully expanded third leaves $9 \mathrm{~h}$ & fastq & $\begin{array}{l}\text { NCBI Sequence Read Archive (http://identifiers.org/ncbi/insdc } \\
\text {.sra:SRR11442889) }\end{array}$ \\
\hline Data file 52 & RNA-seq of BTx623: fully expanded third leaves $12 \mathrm{~h}$ & fastq & $\begin{array}{l}\text { NCBI Sequence Read Archive (http://identifiers.org/ncbi/insdc } \\
\text {.sra:SRR11442888) }\end{array}$ \\
\hline Data file 53 & RNA-seq of BTx623: fully expanded third leaves $15 \mathrm{~h}$ & fastq & $\begin{array}{l}\text { NCBI Sequence Read Archive (http://identifiers.org/ncbi/insdc } \\
\text {.sra:SRR11442887) }\end{array}$ \\
\hline Data file 54 & RNA-seq of BTx623: fully expanded third leaves $18 \mathrm{~h}$ & fastq & $\begin{array}{l}\text { NCBI Sequence Read Archive (http://identifiers.org/ncbi/insdc } \\
\text {.sra:SRR11442886) }\end{array}$ \\
\hline Data file 55 & RNA-seq of BTx623: fully expanded third leaves $21 \mathrm{~h}$ & fastq & $\begin{array}{l}\text { NCBI Sequence Read Archive (http://identifiers.org/ncbi/insdc } \\
\text {.sra:SRR11442884) }\end{array}$ \\
\hline Data file 56 & RNA-seq of BTx623: fully expanded third leaves $24 \mathrm{~h}$ & fastq & $\begin{array}{l}\text { NCBI Sequence Read Archive (http://identifiers.org/ncbi/insdc } \\
\text {.sra:SRR11442883) }\end{array}$ \\
\hline
\end{tabular}


Table 1 (continued)

\begin{tabular}{|c|c|c|c|}
\hline Label & Name of data file/data set & $\begin{array}{l}\text { File types (file } \\
\text { extension) }\end{array}$ & Data repository and identifier (DOI or accession number) \\
\hline Data file 57 & RNA-seq of BTx623: fully expanded third leaves $27 \mathrm{~h}$ & fastq & $\begin{array}{l}\text { NCBI Sequence Read Archive (http://identifiers.org/ncbi/insdc } \\
\text {.sra:SRR11442882) }\end{array}$ \\
\hline Data file 58 & RNA-seq of BTx623: fully expanded third leaves $30 \mathrm{~h}$ & fastq & $\begin{array}{l}\text { NCBI Sequence Read Archive (http://identifiers.org/ncbi/insdc } \\
\text {.sra:SRR11442881) }\end{array}$ \\
\hline Data file 59 & RNA-seq of BTx623: fully expanded third leaves $33 \mathrm{~h}$ & fastq & $\begin{array}{l}\text { NCBI Sequence Read Archive (http://identifiers.org/ncbi/insdc } \\
\text {.sra:SRR11442880) }\end{array}$ \\
\hline Data file 60 & RNA-seq of BTx623: fully expanded third leaves $36 \mathrm{~h}$ & fastq & $\begin{array}{l}\text { NCBI Sequence Read Archive (http://identifiers.org/ncbi/insdc } \\
\text {.sra:SRR11442879) }\end{array}$ \\
\hline Data file 61 & RNA-seq of BTx623: fully expanded third leaves $39 \mathrm{~h}$ & fastq & $\begin{array}{l}\text { NCBI Sequence Read Archive (http://identifiers.org/ncbi/insdc } \\
\text {.sra:SRR11442878) }\end{array}$ \\
\hline Data file 62 & RNA-seq of BTx623: fully expanded third leaves $42 \mathrm{~h}$ & fastq & $\begin{array}{l}\text { NCBI Sequence Read Archive (http://identifiers.org/ncbi/insdc } \\
\text {.sra:SRR11442877) }\end{array}$ \\
\hline Data file 63 & RNA-seq of BTx623: fully expanded third leaves 45 h & fastq & $\begin{array}{l}\text { NCBI Sequence Read Archive (http://identifiers.org/ncbi/insdc } \\
\text {.sra:SRR11442876) }\end{array}$ \\
\hline Data file 64 & RNA-seq of BTx623: fully expanded third leaves $48 \mathrm{~h}$ & fastq & $\begin{array}{l}\text { NCBI Sequence Read Archive (http://identifiers.org/ncbi/insdc } \\
\text {.sra:SRR11442875) }\end{array}$ \\
\hline Data file 65 & RNA-seq of BTx623: fully expanded third leaves $51 \mathrm{~h}$ & fastq & $\begin{array}{l}\text { NCBI Sequence Read Archive (http://identifiers.org/ncbi/insdc } \\
\text {.sra:SRR11442873) }\end{array}$ \\
\hline Data file 66 & RNA-seq of BTx623: fully expanded third leaves $54 \mathrm{~h}$ & fastq & $\begin{array}{l}\text { NCBI Sequence Read Archive (http://identifiers.org/ncbi/insdc } \\
\text {.sra:SRR11442868) }\end{array}$ \\
\hline Data file 67 & RNA-seq of BTx623: fully expanded third leaves $57 \mathrm{~h}$ & fastq & $\begin{array}{l}\text { NCBI Sequence Read Archive (http://identifiers.org/ncbi/insdc } \\
\text {.sra:SRR11442868) }\end{array}$ \\
\hline Data file 68 & RNA-seq of BTx623: fully expanded third leaves $60 \mathrm{~h}$ & fastq & $\begin{array}{l}\text { NCBI Sequence Read Archive (http://identifiers.org/ncbi/insdc } \\
\text {.sra:SRR11442866) }\end{array}$ \\
\hline Data file 69 & RNA-seq of BTx623: fully expanded third leaves $63 \mathrm{~h}$ & fastq & $\begin{array}{l}\text { NCBI Sequence Read Archive (http://identifiers.org/ncbi/insdc } \\
\text {.sra:SRR11442865) }\end{array}$ \\
\hline Data file 70 & RNA-seq of BTx623: fully expanded third leaves $66 \mathrm{~h}$ & fastq & $\begin{array}{l}\text { NCBI Sequence Read Archive (http://identifiers.org/ncbi/insdc } \\
\text {.sra:SRR11442864) }\end{array}$ \\
\hline Data file 71 & RNA-seq of BTx623: fully expanded third leaves $69 \mathrm{~h}$ & fastq & $\begin{array}{l}\text { NCBI Sequence Read Archive (http://identifiers.org/ncbi/insdc } \\
\text {.sra:SRR11442863) }\end{array}$ \\
\hline Data file 72 & RNA-seq of BTx623: fully expanded third leaves $72 \mathrm{~h}$ & fastq & $\begin{array}{l}\text { NCBI Sequence Read Archive (http://identifiers.org/ncbi/insdc } \\
\text {.sra:SRR11442862) }\end{array}$ \\
\hline
\end{tabular}

growth chamber under $12 \mathrm{~h}$ light $\left(440 \mu \mathrm{mol} \mathrm{m} \mathrm{m}^{-1} \mathrm{~s}^{-1}\right.$ of photosynthetically active radiation provided by cool white fluorescent bulbs) and $12 \mathrm{~h}$ darkness for 15 days (sorghum, maize) or 20 days (foxtail millet). Light periods were $26-28{ }^{\circ} \mathrm{C}$ and dark periods were $22{ }^{\circ} \mathrm{C}$. Samples were taken at 3-h intervals for a total of 72-h. Sampling began at $3 \mathrm{~h}$ from when lights were turned on at the beginning of the first collection day. Quality of total RNA extracted from samples was confirmed by Bioanalyzer (Agilent). Strand-specific RNAseq libraries were constructed from mRNA, isolated from total RNA by poly(A) selection, as described previously [2]. Multiplexed libraries were paired-end sequenced with 50 cycles (PE50) by Illumina HiSeq 2500 sequencing at the Illumina Sequencing Genomics Resources Core Facility at Weill Cornell Medical College, Ithaca, NY. The average total reads/mapped reads for sorghum, maize and foxtail millet samples was $21,909,425 / 18,716,318,20,791,418 / 15,401,114$ and $21,482,551 / 20,177,538$, respectively. The 72 demultiplexed FASTQ files from Illumina sequencing were deposited at the National Center for Biotechnology Information (NCBI) Sequence Read Archive under accession number PRJNA616061.

\section{Limitations}

- This is RNA-seq analysis of leaf blade tissue from young plants (15-20 days after planting). Care should be taken in directly extrapolating the transcriptome profiles to other tissues and/or older plants.

- The plants were grown in a growth chamber under cool white fluorescent lighting and under well-controlled temperatures. These conditions should be taken into account when making comparisons to plants grown in the field under natural sunlight and temperature. 
- The goal of the RNA-seq design was to capture transcript levels from expressed genes, not to describe the full range of transcript splice variants.

\section{Abbreviations}

NCBI: National Center for Biotechnology Information; PE50: Paired-end sequenced with 50 cycles.

\section{Acknowledgements}

Not applicable.

\section{Authors' contributions}

$\mathrm{CB}$ performed the experiments. YZ prepared the RNA-seq library. $\mathrm{XL}$ and $\mathrm{CB}$ analyzed the data. FGH and JCS designed the study. All authors read and approved the final manuscript.

\section{Funding}

The funding bodies played no role in the design of the study and collection, analysis, and interpretation of data and in writing the manuscript. X.L.: China Scholarship Council Fellowship. FGH: National Science Foundation award IOS1238048; USDA-ARS CRIS project 2030-21000-039-00D.

\section{Availability of data and materials}

All the data described in this Data note can be freely and openly accessed at the NCBI Sequence Read Archive. All the runs are listed with resolving links in Table 1, and all are gathered together under NCBI Sequence Read Archive study accession SRP254420 [3]. Please see Table 1 and reference Lai et al. [1] for details and links to the data.

Ethics approval and consent to participate Not applicable.

\section{Consent for publication}

Not applicable.

\section{Competing interests}

The authors declare that they have no competing interests.

\section{Author details}

${ }^{1}$ Center for Plant Science Innovation \& Department of Agronomy and Horticulture, University of Nebraska-Lincoln, Lincoln 68588, USA. ${ }^{2}$ College of Agricultural Sciences, Xichang University, Liangshan 615000, China. ${ }^{3}$ Department of Plant \& Microbial Biology, University of California, Berkeley, CA 94720, USA.

${ }^{4}$ Plant Gene Expression Center, USDA-ARS, Albany 94710, USA.

Received: 23 September 2020 Accepted: 24 December 2020 Published online: 14 January 2021

\section{References}

1. Lai X, Bendix C, Yan L, Zhang Y, Schnable JC, Harmon FG. Interspecific analysis of diurnal gene regulation in panicoid grasses identifies known and novel regulatory motifs. BMC Genomics. 2020;21:428. https://doi. org/10.1186/s12864-020-06824-3.

2. Wang L, Si Y, Dedow LK, Shao Y, Liu P, Brutnell TP. A low-cost library construction protocol and data analysis pipeline for illumina-based strandspecific multiplex RNA-seq. PLoS ONE. 2011. https://doi.org/10.1371/ journal.pone.0026426.

3. Lai $X$, et al. Interspecific analysis of diurnal gene regulation in panicoid grasses identifies known and novel regulatory motifs. NCBI Sequence Read Archive. http://identifiers.org/ncbi/insdc.sra:SRP254420 (2020).

\section{Publisher's Note}

Springer Nature remains neutral with regard to jurisdictional claims in published maps and institutional affiliations.
Ready to submit your research? Choose BMC and benefit from:

- fast, convenient online submission

- thorough peer review by experienced researchers in your field

- rapid publication on acceptance

- support for research data, including large and complex data types

- gold Open Access which fosters wider collaboration and increased citations

- maximum visibility for your research: over 100M website views per year

At BMC, research is always in progress.

Learn more biomedcentral.com/submissions 